\title{
Comparing measures of hematologic response after high-dose melphalan and stem cell transplantation in AL amyloidosis
}

\author{
Shayna Sarosiek', Luke Zheng $\mathbb{1}^{2}$, J. Mark Sloan', Karen Quillen', Dina Brauneis ${ }^{1}$ and Vaishali Sanchorawala (i)
}

\begin{abstract}
Hematologic complete response (hemCR) in $\mathrm{AL}$ amyloidosis requires absence of monoclonal protein by immunofixation electrophoreses (IFE) and normal serum free light chain ratio (FLCR). Recent literature suggests that an involved free light chain (iFLC) $<20 \mathrm{mg} / \mathrm{L}$ or difference in free light chains (dFLC) $<10 \mathrm{mg} / \mathrm{L}$ may more accurately predict outcomes after treatment. We evaluated overall survival in 340 patients treated with high-dose melphalan and stem cell transplantation (SCT). Of 305 patients evaluable 6 months after SCT, 90 (30\%) achieved hemCR, 132 (43\%) $\mathrm{dFLC}<10 \mathrm{mg} / \mathrm{L}, 118$ (39\%) iFLC $<20 \mathrm{mg} / \mathrm{L}$, and 176 (58\%) normal FLCR. Of 215 patients without hemCR, 65 (30\%) had $\mathrm{dFLC}<10 \mathrm{mg} / \mathrm{L}$ and 86 (40\%) had normal FLCR. Overall survival (OS) in those achieving $\mathrm{dFLC}<10 \mathrm{mg} / \mathrm{L}$ or normal FLCR without hemCR was inferior to those achieving hemCR ( $p=0.013$ and $p=0.001)$. OS was not significantly different in patients achieving iFLC $<20 \mathrm{mg} / \mathrm{L}$ without hemCR compared with hemCR $(p=0.243)$. Of those with hemCR, OS was not significantly improved if $\mathrm{dFLC}<10 \mathrm{mg} / \mathrm{L}$ was also achieved $(p=0.852)$, but OS was improved for those with hemCR who also attained iFLC $<20 \mathrm{mg} / \mathrm{L}(p=0.009)$. Multivariate analysis demonstrated absence of monoclonal protein in IFE and iFLC $<20 \mathrm{mg} / \mathrm{L}$ as independent predictors of survival. Attainment of hemCR remains a treatment goal, although achieving iFLC $<20 \mathrm{mg} / \mathrm{L}$ may also predict improved OS.
\end{abstract}

\section{Introduction}

Immunoglobulin light chain (AL) amyloidosis is a life threatening hematologic disorder characterized by deposition of amyloid fibrils formed from misfolded and aggregated clonal light chains. Accumulation of amyloid fibrils leads to renal or cardiac dysfunction in the vast majority of patients. Treatment of AL amyloidosis is directed at the underlying clonal plasma or lymphoplasmacytic cells in the bone marrow that are the source of the amyloidogenic light chains. The current criteria used to evaluate hematologic response after treatment were developed in 2012 and validated in patients undergoing high-dose melphalan and stem cell transplantation

\footnotetext{
Correspondence: Vaishali Sanchorawala (Vaishali.sanchorawala@bmc.org) ${ }^{1}$ Amyloidosis Center, Boston University School of Medicine, Stem Cell Transplantation Program in the Section of Hematology and Medical Oncology, Boston Medical Center, Boston, MA, USA

${ }^{2}$ Department of Biostatistics, Boston University School of Public Health, Boston, MA, USA
}

$(\mathrm{HDM} / \mathrm{SCT})^{1,2}$. These criteria define a complete hematologic complete response (hemCR) as the absence of monoclonal protein in serum and urine by immunofixation electrophoresis (IFE) and a normal serum free light chain ratio (FLCR). Very good partial response (VGPR) is defined as a difference in involved and uninvolved serum free light chains $(\mathrm{dFLC})<40 \mathrm{mg} / \mathrm{L}$ and partial response (PR) as a decrease in the dFLC by $>50 \%$ compared to baseline.

After achieving a hematologic response, ideally a hematologic CR (hemCR), a patient is typically monitored to assess for achievement of an organ response. Organ response lags behind hematologic response with renal response occurring on average 7 months after treatment initiation (range, 1-120) and cardiac response at an

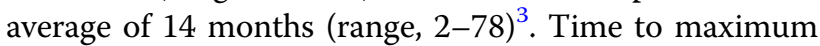
organ improvement may be years after effective treatment. Recent data suggest that organ responses, as well as other patient outcomes, depend on the depth of 
hematologic response. Manwani et al. reported that in a cohort of 915 patients treated with upfront bortezomib, the achievement of a stringent $\mathrm{dFLC}$ response (defined as a $\mathrm{dFLC}<10 \mathrm{mg} / \mathrm{L}$ ) resulted in improved overall survival (OS) and time to next treatment when compared with those patients that did not achieve a dFLC $<10 \mathrm{mg} / \mathrm{L}^{4}$. Additional data also demonstrate that an involved serum free light chain (iFLC) $\leq 20 \mathrm{mg} / \mathrm{L}$ predicts for better OS in patients with $\mathrm{AL}$ amyloidosis who have an underlying lambda plasma cell dyscrasia ${ }^{5}$. Similarly, achievement of a hemCR or VGPR with absence of a monoclonal protein by IFE in combination with a low/normal iFLC is reported to lead to improved progression free survival, longer OS, and higher rates of organ response ${ }^{6}$.

Achievement of a dFLC $<10 \mathrm{mg} / \mathrm{L}$ or an iFLC $<20 \mathrm{mg} / \mathrm{L}$ have not been incorporated into the hematologic response criteria for AL amyloidosis. Moreover, these criteria have not been evaluated in a population of patients treated only with HDM/SCT. This study was designed to evaluate OS in patients with or without a hemCR after HDM/SCT comparing those who achieved an iFLC $<20 \mathrm{mg} / \mathrm{L}$, a $\mathrm{dFLC}<10 \mathrm{mg} / \mathrm{L}$, or a normal FLCR.

\section{Methods}

All patients with AL amyloidosis treated with HDM/ SCT between January 2003 and December 2018 at Boston Medical Center and Boston University Amyloidosis Center were included in this analysis of prospectively collected data. Eligibility for HDM/SCT was based on previously described criteria ${ }^{7}$. Informed consent for prospective data collection was obtained in accordance with the Declaration of Helsinki and the study was approved by the Boston University Medical Center Institutional Review Board. Demographic, clinical, and laboratory data were collected and analyzed for each patient. Hematologic response according to International Society of Amyloidosis validated criteria was assessed at $\sim 6$ months, the typical interval for evaluation after HDM/SCT. Based on the aforementioned criteria, bone marrow examination was not required for assessment of hematologic response. Serum FLCs were quantified with Freelite reagent sets using the Binding Site Optilite analyzer (The Binding Site Ltd) with reference intervals of 3.3-19.4 mg/L for kappa, $5.7-26.3 \mathrm{mg} / \mathrm{L}$ for lambda, and $0.26-1.65$ for the FLCR. Organ involvement was based on Congo red positive staining or clinical criteria ${ }^{1,8}$. Renal staging was based on estimated glomerular filtration rate and proteinuria per established criteria ${ }^{9}$ and cardiac staging was determined by troponin-I and B-type natriuretic peptide per the Boston University cardiac staging system ${ }^{10}$. Kaplan-Meier survival curves were generated to compare survival between patients with hemCR by International Society of Amyloidosis criteria compared with those not achieving a CR but with an iFLC $<20 \mathrm{mg} / \mathrm{L}$, a dFLC $<10 \mathrm{mg} / \mathrm{L}$, or a normal FLCR. In addition, survival was compared in patients achieving a hemCR in addition to an iFLC < $20 \mathrm{mg} / \mathrm{L}$ or a dFLC $<10 \mathrm{mg} / \mathrm{L}$. Survival in patients with an iFLC $<20 \mathrm{mg} / \mathrm{L}$ with and without a hemCR was also compared. Moreover, survival in patients with absence of monoclonal protein on IFE and achievement of either iFLC $<20 \mathrm{mg} / \mathrm{L}$ or dFLC $<10 \mathrm{mg} / \mathrm{L}$ were also compared with patients achieving a hemCR. Hazard ratios (HR) for death and 95\% confidence intervals (CI) were estimated using multivariate Cox proportional hazards regression models. In order to determine which hematologic variable predicts survival, different models were constructed, and their concordance (C-Statistic) and area under the curve (AUC) were performed.

\section{Results}

During the specified 16 years, a total of 340 patients with AL amyloidosis were treated with HDM/SCT. Thirty-five patients $(10 \%)$ were excluded from analysis due to death prior to 6 month assessment after SCT $(n=$ $22,6 \%)$ or lack of follow-up $(n=13,4 \%)$. Of those evaluable at 6 months $(n=305)$, the median age was 57 years and the majority of patients were male. Baseline demographics and clinical characteristics are outlined in Table 1. The hematologic responses of all evaluable patients at 6 months following HDM/SCT were as follows: 90 patients (30\%) with hemCR, 135 (44\%) with VGPR, 35 (12\%) with PR, and 45 (15\%) with no response/progression of disease. Of the evaluable patients, $132(43 \%)$ had a $\mathrm{dFLC}<10 \mathrm{mg} / \mathrm{L}, 118(39 \%)$ had an $\mathrm{iFLC}<20 \mathrm{mg} / \mathrm{L}$, and 176 (58\%) had a normal FLCR. Of the 215 patients without a hemCR, 63 (29\%) had an iFLC < $20 \mathrm{mg} / \mathrm{L}, 65$ (30\%) had a dFLC $<10 \mathrm{mg} / \mathrm{L}$, and $86(40 \%)$ had a normal FLCR. Of the 90 patients that achieved a hemCR, 55 (61\%) achieved an iFLC $<20 \mathrm{mg} / \mathrm{L}$ and $64(71 \%)$ achieved a $\mathrm{dFLC}<10 \mathrm{mg} / \mathrm{L}$. Sixteen patients (5\%) achieved a modified $\mathrm{CR}$, as defined by absence of monoclonal protein on serum and urine IFE with a normal iFLC level but an abnormal FLCR. In addition, 36 patients (12\%) achieved a hematologic CR, but with an iFLC that was $>20 \mathrm{mg} / \mathrm{L}$.

Median follow-up for evaluable patients was 7.5 years. OS was significantly longer in the group that achieved a hemCR compared to those with a dFLC $<10 \mathrm{mg} / \mathrm{L}$ without a hemCR (median OS not reached vs. 10.2 years, $p=$ 0.013) (Fig. 1a). Similarly, those with a normal FLCR without a hemCR had significantly worse OS compared with those with a hemCR (median OS 10.8 years vs. not reached, $p=0.001$ ) (Fig. 1b). OS was similar for patients achieving a hemCR compared to those with an iFLC $<20 \mathrm{mg} / \mathrm{L}$ without a hemCR (median OS not reached for either group, $p=0.241$ ) (Fig. 1c). Of those with a hemCR, OS was not significantly improved if a dFLC $<10 \mathrm{mg} / \mathrm{L}$ was also achieved ( $p=0.852)$ (Fig. 1d), but for those with a hemCR who also attained an iFLC $<20 \mathrm{mg} / \mathrm{L}$ median OS 
Table 1 Baseline patient characteristics.

\begin{tabular}{|c|c|c|c|c|c|}
\hline & \multirow[b]{2}{*}{$\begin{array}{l}\text { Total cohort } \\
(n=305)\end{array}$} & \multirow[b]{2}{*}{$\begin{array}{l}\text { Complete hematologic } \\
\text { response }(n=90)\end{array}$} & \multicolumn{3}{|c|}{ Not achieving hematologic complete response $(n=215)^{a}$} \\
\hline & & & $\begin{array}{l}\mathrm{iFLC}<20 \mathrm{mg} / \mathrm{L} \\
(n=63)\end{array}$ & $\begin{array}{l}\mathrm{dFLC}<10 \mathrm{mg} / \mathrm{L} \\
(n=65)\end{array}$ & $\begin{array}{l}\text { normal FLCR } \\
(n=86)\end{array}$ \\
\hline Median age at time of SCT, range & $57(26-77)$ & $56(26-70)$ & $57(36-74)$ & $58(35-74)$ & $58(35-77)$ \\
\hline Females, $n(\%)$ & $115(38)$ & $36(40)$ & $34(55)$ & $31(48)$ & $31(36)$ \\
\hline \multicolumn{6}{|l|}{ Organ involvement } \\
\hline Cardiac, $n(\%)$ & $160(53)$ & $46(51)$ & $21(34)$ & $26(41)$ & $37(44)$ \\
\hline Renal, $n(\%)$ & $240(79)$ & $74(82)$ & $49(79)$ & $53(83)$ & $78(92)$ \\
\hline Liver, $n(\%)$ & $37(12)$ & $17(19)$ & $6(10)$ & $8(13)$ & $12(14)$ \\
\hline Peripheral nervous system, n (\%) & $32(11)$ & $14(16)$ & $4(7)$ & $5(8)$ & $4(5)$ \\
\hline Autonomic nervous system, $n(\%)$ & $51(17)$ & $16(18)$ & $10(16)$ & $14(22)$ & $15(18)$ \\
\hline \multicolumn{6}{|l|}{ Boston University Cardiac Stage ${ }^{b}$} \\
\hline Stage I, n (\%) & $82(27)$ & $22(24)$ & $26(42)$ & $26(41)$ & $24(28)$ \\
\hline Stage II, $n(\%)$ & $68(22)$ & $19(21)$ & $11(18)$ & $11(17)$ & $15(18)$ \\
\hline Stage III, n (\%) & $26(9)$ & $7(8)$ & $5(8)$ & $5(8)$ & $8(9)$ \\
\hline Stage IIIb, n (\%) & $8(3)$ & $3(3)$ & 0 & $2(3)$ & $3(4)$ \\
\hline \multicolumn{6}{|l|}{ Renal stage ${ }^{c}$} \\
\hline Stage I, n (\%) & $143(47)$ & $33(37)$ & $29(47)$ & $29(45)$ & $30(35)$ \\
\hline Stage $\|, n(\%)$ & $117(38)$ & $37(41)$ & $26(42)$ & $27(42)$ & $36(42)$ \\
\hline Stage III, $n$ (\%) & $42(14)$ & $18(21)$ & $8(13)$ & $9(14)$ & $18(21)$ \\
\hline \multicolumn{6}{|l|}{ Hematologic parameters } \\
\hline Median dFLC, mg/L (range) ${ }^{\mathrm{d}}$ & $79(0-7094)$ & $60(0.3-2282)$ & $28(0.1-879)$ & $28(0.1-771)$ & $36(0.1-1157)$ \\
\hline Bone marrow plasma cell \% (range) & $10(0-50)$ & $10(0-50)$ & $10(0-30)$ & $10(0-30)$ & $10(0-30)$ \\
\hline \multicolumn{6}{|l|}{ Melphalan dose } \\
\hline $100 \mathrm{mg} / \mathrm{m}^{2}, n(\%)$ & $13(4)$ & $2(2)$ & $2(3)$ & $4(6)$ & 7 (8) \\
\hline 140 mg/m², n (\%) & $103(34)$ & $27(30)$ & $17(27)$ & $21(33)$ & $32(37)$ \\
\hline 200 mg/m², n (\%) & $189(62)$ & $61(68)$ & $44(71)$ & $40(63)$ & $47(55)$ \\
\hline
\end{tabular}

SCT stem cell transplantation, FFLC difference between involved and uninvolved free light chains.

${ }^{a}$ Of those without a hematologic complete response, patients may be included in 0 or $\geq 1$ category depending on free light chain results.

${ }^{\mathrm{b}}$ Cardiac staging data unavailable in 121 patients of total cohort.

'Renal staging data unavailable in 3 patients of total cohort.

${ }^{d} \mathrm{dFLC}$ calculations were performed using a value of 0 for all FLC levels reported as less than measurable in 26 patients.

was significantly improved compared to a hemCR alone (median OS not reached for either group, $p=0.009$ ) (Fig. 1e). OS at 10 years was $95 \%$ for those achieving a hemCR with an iFLC $<20 \mathrm{mg} / \mathrm{L}$ and $64 \%$ for those without a hemCR with an iFLC $<20 \mathrm{mg} / \mathrm{L}$ (median survival not reached for either group, $p=0.021$ ) (Fig. 1f). There was no statistically significant difference in OS between those with hemCR and those with $\mathrm{dFLC}<10 \mathrm{mg} / \mathrm{L}$ and absence of monoclonal protein on IFE ( $p=0.747)$ (Fig. $1 \mathrm{~g})$. Similarly, there was no significant difference in OS between those with hemCR and those with iFLC $<20 \mathrm{mg} / \mathrm{L}$ and absence of monoclonal protein on IFE $(p=0.269)$ (Fig. 1h). Of note, the analyses for Fig. $1 \mathrm{~g}$, h are limited due to small sample size.

Multivariate Cox proportional hazard models demonstrate that IFE negativity (absence of monoclonal protein in serum and urine IFEs) and iFLC $<20 \mathrm{mg} / \mathrm{L}$ were independent predictors of survival. In contrast, dFLC $<10 \mathrm{mg} / \mathrm{L}$ was not predictive of survival. Although not statistically significant, our model demonstrates a trend toward improved OS in patients with normal FLCR (HR, 0.590; 95\% CI 0.345-1.007; $p=0.053$ ) (Table 2).

\section{Discussion}

These data demonstrate that achievement of a hemCR using established criteria is superior to achieving only a $\mathrm{dFLC}<10 \mathrm{mg} / \mathrm{L}$ or a normal FLCR for patients undergoing treatment with $\mathrm{HDM} / \mathrm{SCT}$ for $\mathrm{AL}$ amyloidosis. This supports the idea that absence of a monoclonal protein by serum and urine immunofixation electrophoresis is an important part of the hematologic response criteria, as the majority of patients that did not meet criteria for a hemCR had continued presence of monoclonal protein by immunofixation. No significant difference was seen in OS for patients achieving an iFLC $<20 \mathrm{mg} / \mathrm{L}$ compared with a hemCR, despite 27 (44\%) of those with an iFLC $<20 \mathrm{mg} / \mathrm{L}$ having an abnormal FLCR, suggesting that this parameter is likely more predictive than a normal FLCR. These findings are also supported by our multivariate analysis demonstrating independent 
A.

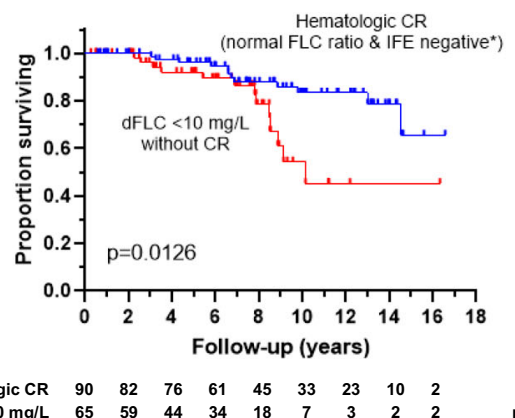

C.

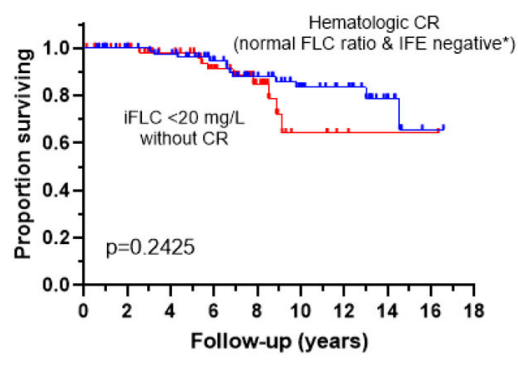
$\begin{array}{ccccccccccccccccccccc}\text { Hematologic CR } & 90 & 81 & 76 & 61 & 45 & 33 & 23 & 10 & 2 & \mathrm{dFLC}<10 \mathrm{mg} / \mathrm{L} & 64 & 62 & 56 & 45 & 32 & 22 & 14 & 5 & \\ \text { ifLC }<20 \mathrm{mg} / \mathrm{L} & 63 & 57 & 48 & 37 & 22 & 7 & 3 & 2 & 2 & \mathrm{dFLC} \geq 10 \mathrm{mg} / \mathrm{L} & 26 & 21 & 21 & 17 & 14 & 12 & 10 & 6 & 2\end{array}$

E.

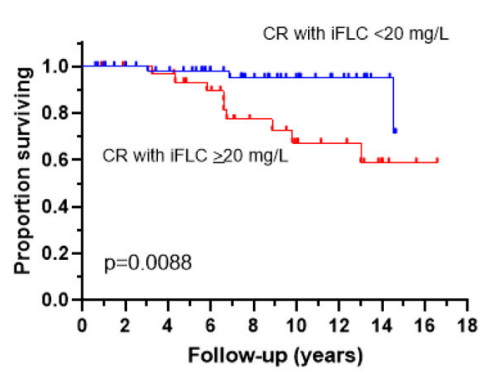

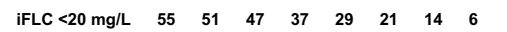

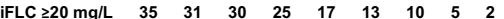

G .

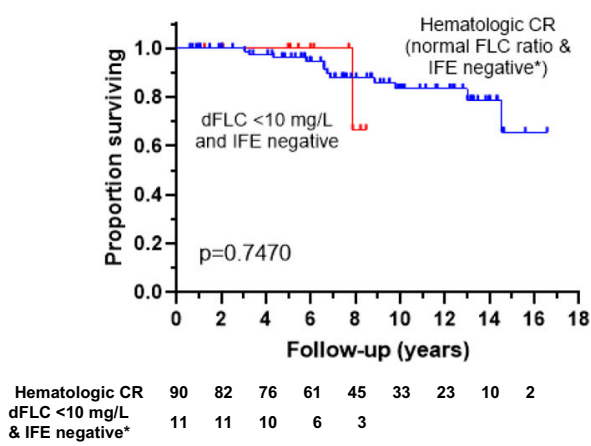

H .

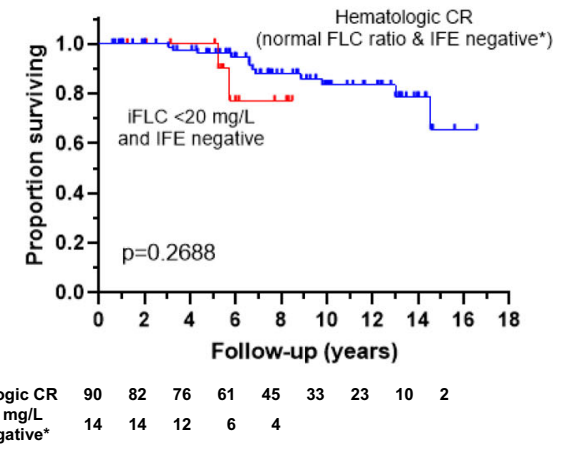

F.

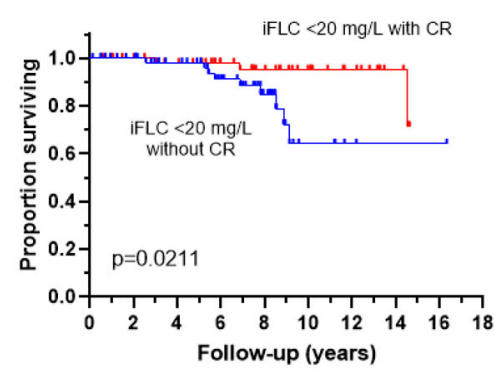

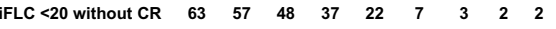

$\begin{array}{lllllllll}\text { ifLC }<20 \text { with } C R & 55 & 51 & 47 & 37 & 29 & 21 & 14 & 6\end{array}$

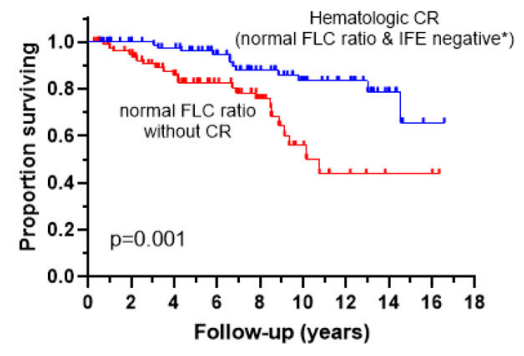

D.
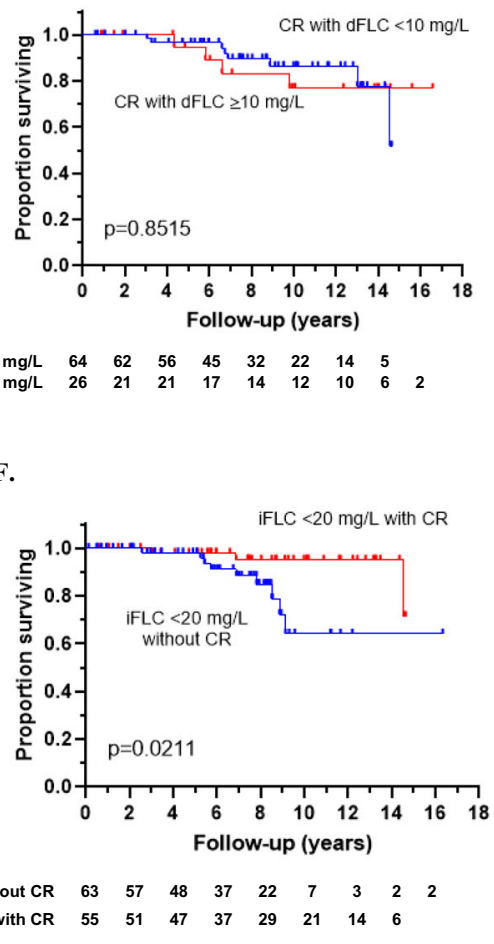

.

Fig. 1 (See legend on next page.) 
(see figure on previous page)

Fig. 1 Overall survival of patients with hematologic complete response (hemCR) versus those without hemCR following treatment with HDM/SCT in AL amyloidosis. a Median overall survival for the patients with hemCR versus dFLC $<10 \mathrm{mg} / \mathrm{L}$ without hemCR ( $p=0.013)$; not reached vs 10.2 years. b Median overall survival for the patients with hemCR versus normal FLCR without hemCR ( $p=0.001)$; Not reached vs 10.8 years. c Median overall survival for the patients with hemCR versus iFLC $<20 \mathrm{mg} / \mathrm{L}$ without hemCR $(p=0.242)$; Not reached for both groups. $\mathbf{d}$ Median overall survival for the patients with hemCR with $\mathrm{dFLC}<10 \mathrm{mg} / \mathrm{L}$ versus hemCR with $\mathrm{dFLC} \geq 10 \mathrm{mg} / \mathrm{L}(p=0.852)$; not reached for both groups. e Median overall survival for the patients with hemCR with $\mathrm{FLLC}<20 \mathrm{mg} / \mathrm{L}$ versus hemCR with iFLC $\geq 20 \mathrm{mg} / \mathrm{L}(p=0.008)$; median OS not reached for both groups. $\mathbf{f}$ Median overall survival for the patients with iFLC $<20 \mathrm{mg} / \mathrm{L}$ with or without hemCR $(p=0.021)$; not reached for both groups. g Median overall survival for the patients with hemCR versus $\mathrm{dFLC}<10 \mathrm{mg} / \mathrm{L}$ and IFE negative $(p=0.747)$; not reached for both groups. $\mathbf{h}$ Median overall survival for the patients with hemCR versus iFLC $<20 \mathrm{mg} / \mathrm{L}$ and IFE negative $(p=0.269)$; not reached for both groups.

Table 2 Multivariate analysis predicting overall survival using different hematologic variables.

\begin{tabular}{|c|c|c|c|c|c|c|}
\hline \multirow[b]{2}{*}{ Variable } & \multicolumn{2}{|c|}{$\begin{array}{l}\text { Model 1: iFLC and dFLC } \\
\text { C-statistic }=0.6575 \\
\text { AUC }=0.6824 \\
n=305\end{array}$} & \multicolumn{2}{|c|}{$\begin{array}{l}\text { Model 2: IFE and FLCR } \\
\text { C-statistic }=0.6973 \\
\text { AUC }=0.7070 \\
n=268\end{array}$} & \multicolumn{2}{|c|}{$\begin{array}{l}\text { Model 3: IFE, iFLC and dFLC } \\
\text { C-statistic }=0.7136 \\
\text { AUC }=0.7354 \\
n=305\end{array}$} \\
\hline & $\mathrm{HR}(95 \% \mathrm{Cl})$ & $p$ value & $\mathrm{HR}(95 \% \mathrm{Cl})$ & $p$ value & HR $(95 \%$ Cl) & $p$ value \\
\hline IFE negative & & & $0.320(0.590,0.637)$ & 0.0012 & $0.374(0.202,0.690)$ & 0.0017 \\
\hline Normal FLCR & & & $0.590(0.345,1.007)$ & 0.0532 & & \\
\hline $\mathrm{iFLC}<20 \mathrm{mg} / \mathrm{L}$ & $0.199(0.093,0.425)$ & $<0.0001$ & & & $0.199(0.092,0.434)$ & $<0.0001$ \\
\hline $\mathrm{dFLC}<10 \mathrm{mg} / \mathrm{L}$ & $1.043(0.562,1.934)$ & 0.8949 & & & $1.528(0.787,2.967)$ & 0.2106 \\
\hline
\end{tabular}

IFE absence of monoclonal protein in serum and urine immunofixation electrophoresis, FLCR free light chain ratio, $i F L C$ involved free light chain level, $d F L C$ difference in involved and uninvolved free light chain level, $\mathrm{C}$-Statistic concordance, $A U C$ area under the curve, $\mathrm{HR}$ hazard ratio, $\mathrm{Cl}$ confidence interval.

Bold denotes statistically significant value.

predictive value of immunofixation electrophoresis and iFLC.

Free light chain levels can be affected by a number of clinical factors, including impaired renal function ${ }^{11}$, underlying inflammatory or infectious conditions ${ }^{12}$, and new monoclonal antibody therapies such as daratumumab and isatuximab ${ }^{13}$. Therefore free light chain measurements may not accurately reflect an individual's hematologic response. Despite this fact, we found that additional improvement in OS is seen in patients with a hemCR who also achieve an iFLC $<20 \mathrm{mg} / \mathrm{L}$. This supports the claim that a maximal FLC response (lower than the standard upper limit of normal for the lambda serum free light chain) may be associated with even better outcomes in those with a hemCR. Newer assessments of hematologic response, such as minimal residual disease measurement by next generation sequencing and multiparametric flow cytometry may be additional manners of measuring deeper hematologic responses ${ }^{14,15}$. Novel modalities that detect monoclonal proteins with higher sensitivity, such as next generation sequencing, multiparametric flow cytometry, matrix-assisted laser desorption ionization time-of-flight, and mass-spectrometry- based assay may also prove to predict patient outcomes $^{16,17}$.

A recent report from the UK National Amyloidosis Center reported that the achievement of $\mathrm{dFLC}<10 \mathrm{mg} / \mathrm{L}$ in a cohort of 915 patients treated with upfront bortezomib resulted in improved OS and time to next treatment when compared with those patients that did not achieve a $\mathrm{dFLC}<10 \mathrm{mg} / \mathrm{L}^{4}$. However, our study failed to show the predictive power of $\mathrm{dFLC}<10 \mathrm{mg} / \mathrm{L}$ with respect to $\mathrm{OS}$ by Kaplan-Meier analysis as well as by multivariate models. This may be related to smaller sample size as well as different treatment regimens as all the patients in our study were treated with HDM/SCT.

This study is limited by its retrospective nature. These findings require additional validation in larger prospective patient cohorts, including patients receiving non-SCT treatment modalities and those with comorbid medical conditions, such as renal failure, which may affect FLC levels. Despite these limitations, our data support the conclusion of recent publications indicating improvement in outcomes in patients with a hemCR (with absent monoclonal protein by immunofixation electrophoresis) and low serum free light chain levels. 
In conclusion, achievement of a hemCR by the established hematologic response criteria (which includes the absence of monoclonal protein on IFE) is superior to achieving only a dFLC $<10 \mathrm{mg} / \mathrm{L}$ or a normal FLCR. The potential inclusion of an iFLC $<20 \mathrm{mg} / \mathrm{L}$, or perhaps other markers of a deeper hematologic response, in addition to serum FLCR normalization may lead to better prediction of $\mathrm{OS}$ in patients with $\mathrm{AL}$ amyloidosis and should be further investigated.

\section{Author contributions}

All authors participated in patient care, and assisted with editing and revision of the paper. SS and VS have written the original paper and analyzed data.

\section{Conflict of interest}

The authors declare that they have no conflict of interest.

\section{Publisher's note}

Springer Nature remains neutral with regard to jurisdictional claims in published maps and institutional affiliations.

Received: 15 May 2020 Revised: 11 August 2020 Accepted: 19 August 2020 Published online: 01 September 2020

\section{References}

1. Palladini, G. et al. New criteria for response to treatment in immunoglobulin light chain amyloidosis based on free light chain measurement and cardiac biomarkers: impact on survival outcomes. J. Clin. Oncol. 30, 4541-4549 (2012).

2. Girnius, S., Seldin, D., Cibeira, M. \& Sanchorawala, V. New hematologic response criteria predict survival in patients with immunoglobulin light chain amyloidosis treated with high-dose melphalan and autologous stem-cell transplantation. J. Clin. Oncol. 31, 2749-2750 (2013).

3. Muchtar, E. et al. Ten-year survivors in AL amyloidosis: characteristics and treatment pattern. Br. J. Haematol. 187, 588-594 (2019).
4. Manwani, R. et al. A prospective observational study of 915 patients with systemic AL amyloidosis treated with upfront bortezomib. Blood 134, 2271-2280 (2019).

5. Muchtar, E. et al. Optimizing deep response assessment for $\mathrm{AL}$ amyloidosis using involved free light chain level at end of therapy: failure of the serum free light chain ratio. Leukemia 33, 527-531 (2019).

6. Sidana, S. et al. Revisiting complete response in light chain amyloidosis. Leukemia 34, 472-1475 (2020).

7. Cibeira, M. et al. Outcome of AL amyloidosis after high-dose melphalan and autologous stem cell transplantation: long-term results in a series of 421 patients. Blood 118, 4346-4352 (2011).

8. Gertz, M. A. et al. Definition of organ involvement and treatment response in immunoglobulin light chain amyloidosis (AL): a consensus opinion from the 10th International Symposium on Amyloid and Amyloidosis. Am. J. Hematol. 79, 319-328 (2005).

9. Palladini, G. et al. A staging system for renal outcome and early markers of renal response to chemotherapy in AL amyloidosis. Blood 124, 2325-2332 (2014).

10. Lilleness, B., Ruberg, F., Mussinelli, R., Doros, G. \& Sanchorawala, V. Development and validation of a survival staging system incorporating BNP in patients with light chain (AL) amyloidosis. Blood 133, 215-233 (2018).

11. Hutchison, C. A. et al. Quantitative assessment of serum and urinary polyclonal free light chains in patients with chronic kidney disease. Clin. J. Am. Soc. Nephrol. 3, 1684-1690 (2008).

12. Piehler, A. P., Gulbrandsen, N., Kierulf, P. \& Urdal, P. Quantitation of serum free light chains in combination with protein electrophoresis and clinical information for diagnosing multiple myeloma in a general hospital population. Clin. Chem. 54, 1823-1830 (2008).

13. Sanchorawala, V. et al. Safety, tolerability, and response rates of daratumumab in relapsed al amyloidosis: results of a Phase II Study. Blood 135, 1541-1547 (2020).

14. Staron, A. et al. Assessment of minimal residual disease using multiparametric flow cytometry in patients with AL amyloidosis. Blood Adv. 4, 880-884 (2020).

15. Muchtar, E. et al. Survival impact of achieving minimal residual negativity by multi-parametric flow cytometry in AL amyloidosis. Amyloid 27, 13-16 (2020).

16. Sharpley, F. A. et al. A novel mass spectrometry method to identify the serum monoclonal light chain component in systemic light chain amyloidosis. Blood Cancer J. 9, 1-4 (2019).

17. Milani, P. et al. The utility of MASS-FIX to detect and monitor monoclonal proteins in the clinic. Am. J. Hematol. 92, 772-779 (2017). 